\title{
A Study of Vision Ergonomic of LED Display Signs on Different Environment Illuminance
}

\author{
Jeih-Jang Liou, Li-Lun Huang, Chih-Fu Wu, Chih-Lung Yeh, \\ and Yung-Hsiang Chen
}

The Graguate Institude of Design Science, Tatung University

\begin{abstract}
The LED (light emitting diode, also referred to as LED) have already been used widely. However, despite the high visibility of LED with high brightness performance, it also leads to a glare problem, which generates a direct security issue in applying to traffics. Therefore, this research aimed to study how to make the LED display sign be more legible under high illuminative environments and to avoid the observers feeling dazzling glare under low illuminative environments. This research firstly studied the literatures to explore the drivers' visual ergonomic as well as the optical properties of LED, and investigated the relatively existing norms for engineering vehicle LED display signs. Three variables were set in this study: three kinds of ambient illumination, four kinds of luminance contrast and two kinds of character form. In the first phase of the experiment, subjects observed LED display signs in both near and distant locations and filled out the SWN scale (Subjective Well-being under Neuroleptics), and in the second phase, subjects were then asked to moved forward and recorded their perceptions of comfort and glare to distance range. The findings demonstrated that, there was no variation in subjective evaluation to display signs with no backgrounds either in the near or distant locations, while to display signs with backgrounds, the subjects perceptions were the farther the distance, the clearer the legibility; higher ambient illumination could effectively reduce observers' glare perception to LED display signs; display signs with backgrounds at the luminance contrast of 3:1 $\left(\mathrm{L} \max =3100, \mathrm{~L} \min =1033 \mathrm{~cd} / \mathrm{m}^{2}\right)$ showed the lowest uncomfortable and glare level to observers. The two forms of character showed no significant variation in affecting observers in terms of the comfort and glare perception.
\end{abstract}

Keywords: LED display signs, engineering vehicles, legibility, ambient illumination, luminance contrast.

\section{Introduction}

LED, a light source made from the semiconductor technology, has been widely used in IT products, communication electronics, display panel, traffic signal, and various instrumental displays. Owing to the price drop and improving product features, HighBrightness LED has gradually replaced the traditional LED, in addition, as the emerging markets have used High-Brightness LED directly, the traditional LED is now only partly utilized in signs, lighting, electronic equipments etc., and its market has been extremely shrinked. 
Currently, High-Brightness LEDs are mainly applied to mobile phones, displays, automotives, lightings, signal lights, and other areas. In viewing the application and future forecast, the DisplaySearch pointed out that the outdoor display has been ranked in the top two demands of High-Brightness LED. Therefore, the LED display immediately provides important and dynamic traffic information to people. According to current experience of LED displays application, in order to obtain an ideal display effect outdoor, the brightness has to be over $4000 \mathrm{~cd} / \mathrm{m}^{2}$ [1]; However, in the night, such high brightness might make the observers feel discomfortable and glare. Take the arrow direction traffic light on the rear of national highway engineering vehicle for example, according to the statistics of National Freeway Bureau, there is about six constructional fatal accidents per year, in which three of them usually occurred during the operation of mobile engineering trucks. Although those accidents were not cause by engineering trucks, all seemed to be relevant to the insufficiency of warning signs on the vehicles and related constructional spots, which also showed a high rate of car accident on the highway constructional operation. Therefore, this research aimed to study how to make the brightness of LED display sign on engineering trucks to reach the most comfortable visual distance which allows drivers to have more time to do proper reactions and judgments, as well as avoid causing glare to drivers when they are driving at close range.

Kazunori Munehiro et al. [2] study pointed out that the legible distance of LED guide light in the daytime is father than road marking, while in the mist the glare level will rise with the increased brightness of LED. As this is the situation, an appropriate change of brightness of LED is necessary. Therefore, installing LED on road traffic does improve legibility of drivers. The study of Uchida Kazuhiro et al [3] has indicated that the low legibility during the night is because of the high contrast brought out by too high brightness, while the text and background within a certain contrast can enhance the effect of visual recognition.

On aspect of the application of LED display signs in each country, according to the Highway Construction Traffic Control Manual of Directorate General of Highways, MOTC [4], there are at least four levels of the brightness of LED display signs in which the darkest level must be the half of the brightest, and the intensity of each LED light must not be less than $2 \mathrm{~cd}$. .In the manual of the road construction traffic control and safety equipment of Macao Special Administrative Region Transport Bureau [5], it also mentions that the brightness of LED light on the engineering vehicle should be adjusted in accordance with the surrounding illumination. Japan only stipulates two levels of adjustment for the brightness of LED display signs, dividing into day and night luminance in accordance with the difference of color.

From the various regulations for variable message sign and LED signal on engineering vehicles set by each county, it shows that currently there is no uniform provision to the brightness of LED application on engineering truck. Therefore, this research focused on the brightness contrast background between LED variable message sign and LED display panel, conducting the study of visibility under different illumination environments, to investigate how the luminance contrast of LED display sign reached the farthest visible distance under different illumination environments and without creating glare to drivers in close distance. 


\section{Research Methods and Experimental Design}

\subsection{Research Methods}

The purpose of this study was to explore:

(1) The farthest and comfortable distance of the luminance contrast of LED display sign under different ambient illuminances.

(2) The effect of the character form of LED display signs to subjects' legibility.

\section{Experimental Variables}

(1) The luminance contrast (Lc) of LED sign

This experiment used dimmer to control the different brightnesses of LED and LP9221 UNM6 Luminometer to measure the luminance. The luminance contrast between sign and background was divided into four levels, $\mathrm{Lc} 1\left(\mathrm{~L} \max =6200 \mathrm{~cd} / \mathrm{m}^{2}, \quad \mathrm{~L}\right.$ $\left.\min =0 \mathrm{~cd} / \mathrm{m}^{2}\right) 、 \operatorname{Lc} 2\left(\mathrm{~L} \max =6200 \mathrm{~cd} / \mathrm{m}^{2}, \quad \operatorname{Lmin}=3100 \mathrm{~cd} / \mathrm{m}^{2}\right) 、 \mathrm{Lc} 3(\mathrm{~L} \max =6200 \mathrm{~cd} /$ $\left.\mathrm{m}^{2}, \quad \mathrm{~L} \min =2066 \mathrm{~cd} / \mathrm{m}^{2}\right) 、 \operatorname{Lc} 4\left(\mathrm{~L} \max =3100 \mathrm{~cd} / \mathrm{m}^{2}, \quad \mathrm{~L} \min =1033 \mathrm{~cd} / \mathrm{m}^{2}\right)$.

(2) Ambient illumination

This experiment was conducted at a built darkroom in the third floor of the Department of Industrial Design of Tatung University, and simulated the illumination environments around the clock by using three halogen lamps, in which one lamp was connected to dimmer to control the illumination. The variables of environment illumination were set for three classes, including the bright day (30000 Lux), the dark day (5000 Lux) and night (10Lux). LP 9221 S1 Illuminometer was used to measure the illuminance.

(3) Character exhibition

According to the highway construction traffic control manual of Directorate General of Highways, the main directional contents of advance warning arrow sign on engineering warning car are " $\leftarrow-"$ and $"<<<"$ two styles. The experimental fetched the front part of the arrow signals of " $\leftarrow$ " and " $<"$ (Figure 1) to proceed the experiment of the interaction between the two characters. And the sign appeared randomly from four different directions of up, down, left and right to avoid the subjects' learning effect.

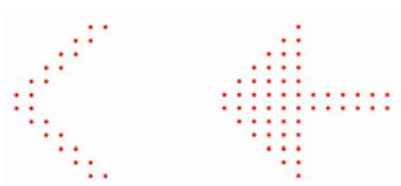

Fig. 1. The characters of arrow sign on LED display panel

\section{Dependent variables}

(1) Subjective assessment questionnaire: a questionnaire for the subjects to assess their eyes comfort in observing the LED signs at the nearest distance (9.8M) and the most far distant (57M). Questionnaire items were: 1. I feel eye fatigue, 2. I 
think things look difficult, 3 . There is a strange feeling around the eyes, 4 . I feel numb, 5. I feel headache 6. I feel dizzy 7. This experimental signal is easy to identify, and the five-point scale was applied with point 1 to 5 which represented from "strongly disagree, disagree, fair, agree, strongly agree.", respectively. Items from1 to 6 were negative visual feelings, item 7 was positive feelings to explore the identification degree to sign.

(2) Range of comfort: the distance which subjects feel comfortable while observing the LED display panels - the distance which subject feel the glare while observing the LED display panels = comfort.

(3) Glare range: the distance which subjects feel glare while observing the LED display panel - point of origin $=$ glare range.

\subsection{Experimental Equipment}

According to the contents of highway construction under traffic control manual, the size of exhibition sign should be in $75 \mathrm{~cm}$ (L) X $150 \mathrm{~cm}(\mathrm{~W})$; however, due to the limitation of experimental space, the size ratio of the sign in this study was reduced to the size of $30 \mathrm{~cm}(\mathrm{~L}) \times 60 \mathrm{~cm}(\mathrm{~W})$, within a total of $16 \times 32$ LED lights.

To brightness control, in order to adjust the brightness difference between the middle section (12 x12 lights) of LED display panel and background, the signal part was connected with controller and each LED of $12 \times 12$ light points was connected to the programmable controller and LUZ-5 relay. The electronic control of signal part was shown in Figure 2.

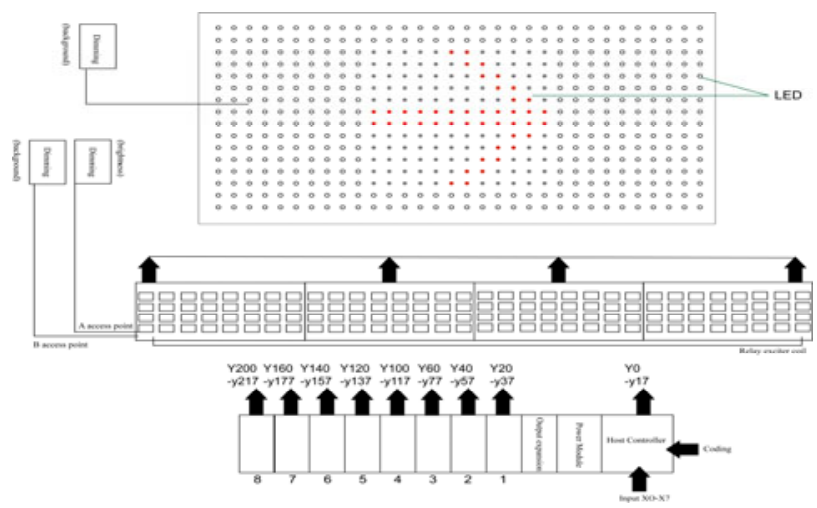

Fig. 2. The diagram of control system of signal section

\subsection{Subjects}

Before experiment proceeding, this research carried out a visual acuity test to all of the subjects in accordance with the standards of obtaining the automobile driving license specified by the Directorate General of Highways M.O.T.C, that is, the naked vision value of both eyes have to be 0.6 or over and each eye has to be over 0.5 or 0.8 after correction, as well as with field of each eye view reaches 150 degree, and without night blindness as well as also be able to distinguish among red ,yellow and 
green colors. Based on Tatung University students as sample population, this study randomly selected 29 subjects ( 15 males, 14 females, aged between 21 to 30 years), while the difference of gender and age were not considered in this experiment.

\subsection{Experimental procedure}

(1) Subjects firstly filled in the basic personnel information in the classroom and their visual acuity values were confirmed to meet the requirement of this experiment. Next, the researcher presented the experiment details to subjects and explained questions raised by subjects.

(2) Subjects sat in chairs with the same height as the car seat and, under the condition of 2 level of character $\mathrm{x} 4$ level of luminance contrast $\mathrm{x} 3$ level of ambient illuminance of LED display sign luminance and ambient illuminance adjusted by the researcher, observed the LED display panel in both of the nearest distance of 9.8 meters and the farthest distance of 57 meters (Figure 3), and then filled out the subjective assessment questionnaire of their perceptions.

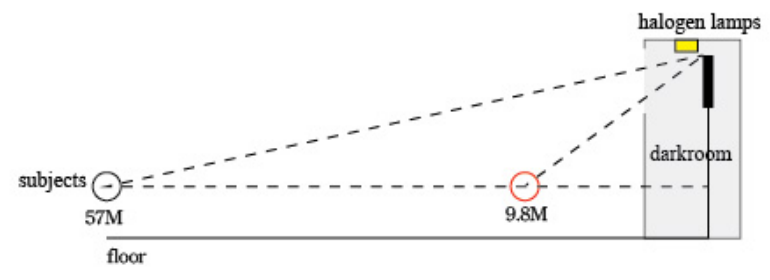

Fig. 3. First phase of experiment

(3) In the second phase, the subjects moved forward slowly from the distance of 57 meters until the they felt comfortable with arrow sign of the LED display panel sand recorded the visual distance at that moment(Figure 4). Then the subjects kept on moving forward until they felt glare to the arrow sign of LED display panel, and again recorded the visual distance at that moment.

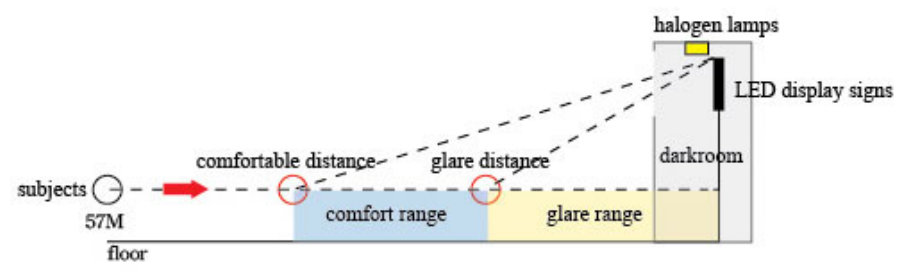

Fig. 4. Second phase of experiment

(4) By the end of each test, the subjects took a short rest to avoid the visual fatigue or discomfort. 
(5) Finally, this research applied Microsoft Excel 2007 to calculate the descriptive statistics for all variables of the experimental results in the first stage. The results of second experimental stage were calculated by SPSS 18.0 software to analyze the interaction between the three factors, and if there is not interaction, the One Way ANOVA analysis would be conducted to explore the variation between single-factor variables.

\section{Results and Discussion}

\subsection{The Descriptive Statistics for Subjective Assessment of the Far and Near Distances}

The subjective assessments were the assessments of different variables combination of 24 groups at the distance of 9.8 meters and 56 meters. The descriptive statistical analysis of $\mathrm{A} \sim \mathrm{X}$ groups was shown in Figure 5.

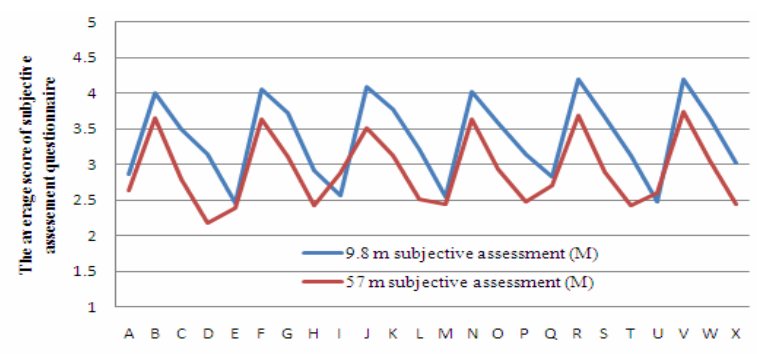

Fig. 5. The subjective assessment of the distances of $9.8 \mathrm{~m}$ and $57 \mathrm{~m}$

Figure 5 showed that, in general, the visual sense in $9.8 \mathrm{~m}$ was inferior to $57 \mathrm{~m}$, only the subjective assessments of A, E, I, M, Q, U groups in different distances showed no significant variation, some of them even appeared a phenomenon of the visual sense in $9.8 \mathrm{~m}$ was superior to $57 \mathrm{~m}$. As shown in Table 1, the statistical visibility compiling from item 7 showed that the variation of average scale of visibility in these six group were less obvious than other groups.

Table 1. The subjective assessment of visibility of A, E, I, M, Q, U groups

\begin{tabular}{|l|l|l|l|l|}
\hline \multirow{2}{*}{ Group } & $9.8 \mathrm{~m}$ subjective assessment of visibility & $57 \mathrm{~m}$ subjective assessment of visibility \\
\cline { 2 - 5 } & Mean & Standard Deviation & Mean & Standard Deviation \\
\hline A & 3.76 & .831 & 3.80 & .957 \\
E & 4.36 & .569 & 4.04 & .935 \\
I & 4.36 & .757 & 3.84 & 1.028 \\
M & 4.32 & .748 & 4.36 & .860 \\
Q & 4.04 & 1.060 & 4.04 & .790 \\
U & 4.20 & .866 & 3.96 & .935 \\
\hline
\end{tabular}


This demonstrated that, either in the near or far distances, there was no variation of the discomfort generated by LED display sign without background; while to LED display sign with background, the subjective experience of observing from far distance was superior to LED display signs without background. This finding was in consistent with the conclusion of the study of Uchida Kazuhiro et al. [3] which suggested that, under a certain contrast range, the character and background have the effect of enhancing the legibility.

\subsection{Comparison of Three-Factor Multivariate Analysis}

The second phase of this research was to conduct experiment for the combination of 3 different variables. In this experiment, the subjects were asked to move forward from the distance of 57 meters to the distance until they felt comfortable to the light source of LED display panel, and then kept on moving forward until to the distance they felt glaze. The subtraction of both distances was the subjects' comfortable range to their variables combination. In order to understand the significance of variation of ambient illumination, LED luminance contrast and character to the perception of comfort and glare, this research used SPSS18.0 to conduct the three-factor multivariate analysis for collected data, the results showed that, in a comfortable distance range, there were $\mathrm{P}=0.994$, and no significant variation in interaction $(\mathrm{P}>0.05)$. In the distance range of glare, the analyzing result showed $\mathrm{P}=0.975$, and no significant variation in interaction $(\mathrm{P}>0.05)$. To those insignificant variations, the reason we inferred was that, normally the ambient illumination at noon is up to $50000 \sim 100000$ Lux, while our experimental environment illumination was just 30000Lux.

The interaction between ambient illumination and character form in the range of comfortable distance and the range of glare were significant different with $\mathrm{P}=0.006$ and $\mathrm{P}=0.004(\mathrm{P}<0.05)$, respectively. The figure 6 and 7 demonstrated that under the 30000 Lux environmental illumination, the range of comfortable distance of arrow sign with tail" $\longleftarrow$ " was farther than arrow sign without tail " $<$ ", and the range of glare distance was shorter in arrow sign with tail. It was speculated that under the higher

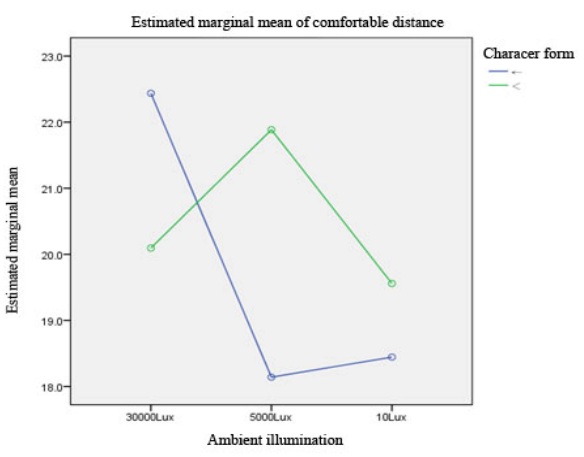

Fig. 6. The comparison of comfortable distances between ambient illumination and character form 
ambient illumination, the LED with more display signs offered greater visual effects; while under lower ambient illumination, such as in the evening or night, the glare generated by the LED with more display signs was higher. The interaction between ambient illumination and luminance contrast to the range of comfortable distance and the range of glare distance demonstrated no significant variation with $\mathrm{P}=0.772$ and $\mathrm{P}$ $=0.734(\mathrm{P}>0.05)$, respectively. The variation between character form and luminance contrast to the range of comfortable distance and the range of glare distance was also not significant with $\mathrm{P}=0.940$ and $\mathrm{P}=0.759(\mathrm{P}>0.05)$, respectively.

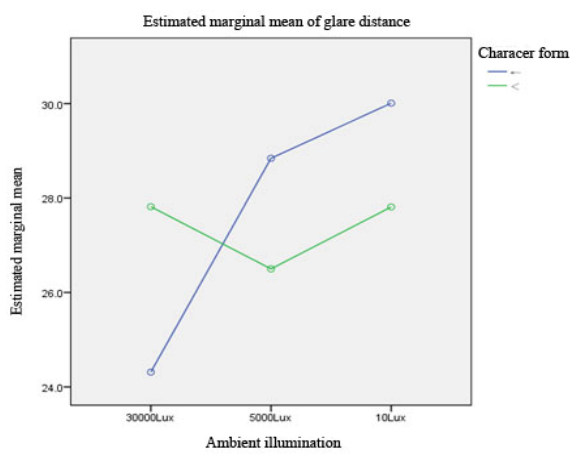

Fig. 7. The comparison of glare distances between ambient illumination and character form

\subsection{The Comparison of One-Way ANOVA}

Finally, this research preceded the LSD (least significant difference) post hoc analysis to ambient illumination and luminance contrast to explore whether there were variations between the levels of various single factors. The results of individual multiple comparisons were as follows:

(1) The comparison of one-way ANOVA of the ambient illumination

The results showed that, under the environment of 30000 Lux and 10 Lux, the glare distance to observer was significant $(\mathrm{P}<0.05)$. The glare distance of ambient illumination at 30000 Lux was short than 10 Lux, which meant that observing under higher ambient illumination would reduce glare extend.

(2) The comparison of one-way ANOVA of Luminance contrast

The LSD multiple comparison of luminance contrast demonstrated that, except Lc1 $(\mathrm{Lmax}=6200, \mathrm{Lmin}=0)$ and $\mathrm{Lc}=3(\mathrm{Lmax}=6200, \mathrm{Lmin}=2066)$, all variations between groups were significant $(\mathrm{P}<0.05)$. The condition of Lc4 $(\mathrm{Lmax}=3100, \mathrm{Lmin}=1033)$ showed the highest comfort and lowest glare compare to the other groups. Lc3 (Lmax=6200, Lmin=2066) was the second best condition in this analysis. The most uncomfortable and glare condition to subjects was Lc2 $(\operatorname{Lmax}=6200$, Lmin $=3100)$. The results showed that the comfortable extend of LED display signs with background were better than those of without background. This result was in line with Uchida Kazuhiro et al [3] finding which concluded that the background brightness can indeed reduce the glare extends generated by the brightness of signs, and lead the observers feel more comfortable. 


\section{(3) The comparison of one-way ANOVA of character form}

The results showed that there was no significant variation in the comfortable and glare distances $(\mathrm{P}>0.05)$, which indicated that the comfortable and glare distance of subjects' observation to the two character forms were not affected. This represented that there was no difference in observing both of the two character forms applied on the LED of Taiwan engineering vehicles.

\section{Conclusion}

With the advance of packaging process technology, the controllable factors of luminous efficiency, brightness of LED have also improved significantly. While in pursing the technologic performance as well as applying LED for human observation, it is necessary to take visual ergonomics into consideration. This study investigated whether environmental light illumination would affect the brightness performance of LED display signs. The results of the experimental analysis of this research were as follows:

(1) The distance of LED display sign with background would affect the observer's viewing comfort. In the visible range, the subjective viewing comfort of farther distance was superior to short distance. At the condition of without background $(\operatorname{Lmax}=6200, \operatorname{Lmin}=0)$ conditions, there was no variation of the subjective comfort between observing the LED display panel from the farther or short distance.

(2) The perceived glare extent of observing the LED display sign in the condition of higher ambient light (30000Lux) was lower than the glare extent in the night; on the contrary, subject's viewing comfort in the environment with higher light (30000Lux) was superior to the viewing comfort in the night (10Lux), which indicated that the higher environmental light source around the LED display signs, the lower glare extent the observer perceived.

(3) In the aspect of character form, there was no significant variation between the two character forms in Taiwan engineering vehicle in terms of the extent of comfort and glare. However, in the condition of the interaction with environmental light in 30000Lux, the comfort and glare extent of arrow sign with tail " $\leftarrow$ " was better than the arrow sign without tail " $<"$; however, in the night (10Lux), the above situations were contrary. It was speculated that the ambient light of 30000Lux reduced its value of glare which led the arrow signal with tail to look more comfortable; while in the condition of 10Lux, the perception of less comfort might be because there were more LED lighting points on the arrow sign with tail " $\leftarrow$ " which resulted the high extent of glare.

(4) From the view point of luminance contrast, similar to LED sign with background on Japan engineering vehicles, the most comfortable luminance contrast of 3:1 was consistent with the results obtained by ANSI / HFS 100 -1988 (1988) recommending the VDT brightness contrast should be 3 at least, while too high brightness will make the observer feel uncomfortable and glare. When Lc $=0$, the perception of uncomfortable and glare will be higher than $\mathrm{Lc}=3$, this finding was also in line with the report of enhancing the night visibility of LED sign device provided by Japan Information Processing Juridical Association A which suggested the way of lighting background can prevent the dazzling glare phenomena. 


\subsection{The Direction of Sequential Studies}

(1) The maximum ambient illumination in the experiment of this research was only up to 30000Lux, but the general illumination in the noon is more high at about $50000 \sim 100000$ Lux, therefore, the ambient illumination should be increased in the sequential studies.

(2) Conduct the experiment in real environment and real size of LED display sign to increase the accuracy.

(3) Include the visibility in the rainfall condition into experiment in accordance with the Taiwan's climatic condition.

(4) Set and explore more luminance contrasts in the experiment in order to obtain the best visibility and comfort.

(5) Target to more character forms to explore different arrow signs (only two character sign in this research).

(6) Aesthetic design should be considered in depth study while in exploring the effective performance of character form.

(7) The future study should consider the legibility of different LED color which was not addressed in this research.

(8) Add the variable of lights flicker frequency to investigate the legible performance.

\section{References}

1. LED Industry network (LEDinside) (2007), http: / / www . ledinside.com. tw/

2. Munehiro, K., et al.: The Monthly Report of Hokkaido Civil Development Institute, No.630 (November 2005)

3. Kazuhiro, U., et al.: Information Processing Society Foundation Research Report, IPSJ (2005)

4. Highway Construction Traffic Control Manual of Directorate General of Highways, MOTC (February 2010)

5. The manual of the road construction traffic control and safety equipment of Macao Special Administrative Region Transport Bureau (January 2009)

6. DisplaySearch, http: //www. displaysearch. com.tw/default.aspx 